\title{
A Big Quantum Computer
}

\author{
Quantum supremacy using a programmable superconducting processor \\ Authors: Frank Arute, Kunal Arya, ... (73 more authors) ... Hartmut Neven, and \\ John Martinis \\ Nature 574, 505-510 (2019)

\section{Recommended with a Commentary by David DiVincenzo, Forschungszentrum Jülich}

It is only possible to touch on a smattering of the content of this paper, the work of 77 scientists, reporting many years of concerted effort. I will look at this through the filter of the condensed matter physicist, and try to pick out some of the things that have been achieved from that point of view, and what it indicates for the future. Something like $80 \%$ of the literary content of this work is contained in a massive supplementary text - 66 pages, 50 figures, 11 tables. Most of what I will mention, most of the condensed matter physics, will be found compressed into there.

The reader can go elsewhere for commentaries about what has been achieved computationally. A device of 53 qubits generates, in a few minutes of operation, samples from a probability distribution, whose generation on a supercomputer will take 10,000 years (or maybe a couple of days, according the claim of Pednault et al., arXiv:1910.09534). So the quantum device is supreme, at least for the problem whose solution nobody was asking for. The road to computations for the real world still stretches out into the future, apparently many more years of work still lie ahead.

I think that the main message for the physicist here is Anderson's: More is Different. The 53 working individual superconducting devices comprising the qubits (out of 54 in the layout) are basically the same transmons that we have had for fifteen years now: capacitively shunted Josephson junctions whose dynamics is that of a moderately anharmonic oscillator. There are an additional 88 transmons along the "bonds" connecting pairs of qubits, and they are coupled in such a way that they control the effective strength of interaction between the qubits - they are the switches. For the qubit transmons, the two lowest energy levels define the 0 and 1 . The transmons are very weakly damped, but they are short of the world record for transmons by a factor of 10 or so, with $T_{1}$ times in the $15 \mu \mathrm{sec}$ range (Fig. S22). But excellent control of the dynamics is achieved by "running fast", that is, all gates, both single qubit and entanging two-qubit gates, occur in times of just $12 \mathrm{nsec}$.

On this chip, a two-dimensional lattice of two-level systems, with extensive control of their quantum dynamics, is achieved. The two-qubit gates are not the conventional controlledNOT of quantum logic theory, but a kind of iSWAP, in which there is "flip flop" interaction, that is rotation between the 01 and 10 states. This is basically the XY interaction of statistical physics, but there are additional, somewhat variable quantum phases (Fig. S14). This 
is $\mathrm{OK}$ in the current application, since the capacity for maximal entanglement is surprisingly generic - in a particular mathematical sense, exactly half of all possible two-qubit evolutions can produce maximal entanglement (Zhang et al., Phys.Rev. A67, 042313 (2003)). Programming a real algorithm would be more demanding, because achieving a standard gate, with phases always the same, would be necessary, and would add overhead.

The parameters of the 53 qubits have been painstakingly documented, and $100 \%$ of them function very well: their frequencies are controlled dynamically to four significant figures, and the fidelity of all quantum operations is around $99 \%$ - this is, loosely speaking, the inner product of the actual quantum state with the ideal entangled quantum state, at the two-qubit level.

The purpose for which this magnificent piece of hardware is used is not condensed matter physics, but computational science. But it is perhaps interesting to ask what it could mean as a future tool for condensed matter physics. I have already mentioned that it provides a patch of a square spin lattice, in the form of a kind of fully instrumented quantum metamaterial. I don't think that anyone has thought through what sort of quantum statistical physics could be done with it. An alternative point of view is to note that this device is a successor to those of quantum transport, which gave us phenomena like Coulomb blockade, Bloch oscillations, quantized Hall effects, and weak localization. These were first done with twoterminal devices; the move to four terminals significantly increased the detail with which these phenomena could be examined. The device in the current experiment constitutes a quantum transport experiment with hundreds of terminals. I think that we have hardly begun to develop the paradigms that will permit us to use this device to uncover new quantum device physics. 